\title{
Review Specialist
}

National Cancer Institute

\section{Source}

National Cancer Institute. Review Specialist. NCI Thesaurus. Code C51866.

An individual with expertise in one or more scientific disciples that can evaluate and review scientific data and funding proposals. 